QUARTERLY OF APPLIED MATHEMATICS

VOLUME LXIV, NUMBER 3

SEPTEMBER 2006, PAGES 413-431

$\mathrm{S}$ 0033-569X(06)01021-1

Article electronically published on June 12, 2006

\title{
ON A TWO-POINT FREE BOUNDARY PROBLEM
}

\author{
BY \\ JONG-SHENQ GUO (Department of Mathematics, National Taiwan Normal University, S-4 Ting \\ Chou Road, Taipei 117, Taiwan) \\ AND \\ BEI HU (Department of Mathematics, University of Notre Dame, Room 255, Hurley, Notre Dame, \\ Indiana 46556)

\begin{abstract}
We study a two-point free boundary problem for a quasilinear parabolic equation. This problem arises in the model of flame propagation in combustion theory. It also arises in the study of the motion of interface moving with curvature in which the studied problem is confined in the conical region bounded by two straight lines and the interface has prescribed touching angles with these two straight lines. Depending on these two touching angles, there are three different cases, namely, area-expanding, area-preserving, and area-shrinking cases. We first give a proof of the global existence in the expanding and preserving cases. Then the convergence to a line in the preserving case is derived. Finally, in the shrinking case, we show the finite-time vanishing and the convergence of the solution to a self-similar solution.
\end{abstract}

1. Introduction. We consider the following two-point free boundary problem (FBP):

$$
\begin{aligned}
& u_{t}=\left[a\left(u_{x}\right)\right]_{x}, \quad-\xi_{1}(t)<x<\xi_{2}(t), t>0, \\
& u_{x}\left(-\xi_{1}(t), t\right)=\tan \alpha_{1}, u_{x}\left(\xi_{2}(t), t\right)=\tan \alpha_{2}, t>0, \\
& u\left(-\xi_{1}(t), t\right)=\xi_{1}(t) \tan \beta_{1}, u\left(\xi_{2}(t), t\right)=\xi_{2}(t) \tan \beta_{2}, t>0, \\
& u(x, 0)=u_{0}(x),-\xi_{1}(0) \leq x \leq \xi_{2}(0), \quad \xi_{1}(0)=\xi_{01}, \quad \xi_{2}(0)=\xi_{02},
\end{aligned}
$$

where we assume that

$$
a \in C^{2}(-\infty, \infty), \quad a(0)=0, a^{\prime}(s)>0 \quad \text { for } s \in(-\infty, \infty),
$$

$\beta_{i}$ and $\alpha_{i}$ are given constants satisfying

$$
\beta_{i} \in(0, \pi / 2), \quad i=1,2, \quad \alpha_{1} \in\left(-\beta_{1}, \pi / 2\right), \quad \alpha_{2} \in\left(-\pi / 2, \beta_{2}\right),
$$

Received January 18, 2005.

2000 Mathematics Subject Classification. Primary 35K20, 35K55.

E-mail address: jsguo@math.ntnu.edu.tw

E-mail address: b1hu@nd.edu 


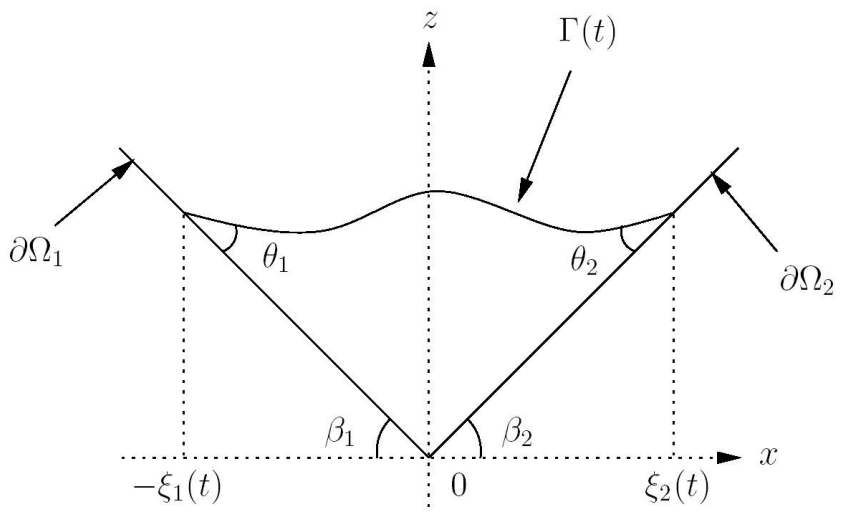

FIG. 1. $\theta_{1}=\alpha_{1}+\beta_{1}>0, \theta_{2}=\beta_{2}-\alpha_{2}>0$

and $\xi_{01}$ and $\xi_{02}$ are positive constants such that

$$
\begin{gathered}
u_{0} \in C^{1+\alpha}\left[-\xi_{01}, \xi_{02}\right], \quad u_{0}\left(-\xi_{01}\right)=\xi_{01} \tan \beta_{1}, \quad u_{0}\left(\xi_{02}\right)=\xi_{01} \tan \beta_{2}, \\
u_{0}(x)>0 \text { for } \xi_{01}<x<\xi_{02} .
\end{gathered}
$$

In this problem, $u, \xi_{1}, \xi_{2}$ are unknown functions to be found.

Figure 1 shows the configuration of our problem, where $\Gamma(t)$ represents the surface $z=u(x, t)$, and the free boundaries are located at $x=-\xi_{1}(t)$ and $x=\xi_{2}(t)$.

This type of free boundary problem arises in various applications. For example, when $a(s)=s$ (the case of the heat equation), (FBP) arises in the model of flame propagation in combustion theory (cf. [1, 9, 12] and the references cited therein). When $a(s)=\arctan (s)$ (the case of the curvature flow equation), (FBP) arises in the study of the motion of the interface moving with curvature in which the studied problem is confined in the conical region bounded by two straight lines and the interface has prescribed touching angles with these two straight lines (cf. 4] and the references cited therein).

There has been much interesting work on the so-called one-point free boundary problem (when $\beta_{1}=0$ and $\beta_{2}=\pi / 2$ ). For the case with $\alpha_{1}=\pi / 4$ and $\alpha_{2}=0$, we refer the readers to [10, 2, 12, 6]. We note that, by a reflection, this case is the symmetric case of our two-point free boundary problem with $\beta_{1}=\beta_{2}=0$ and $-\alpha_{2}=\alpha_{1}=\pi / 4$. For the case with $\alpha_{1}, \alpha_{2} \in(0, \pi / 2)$, we refer the readers to [11].

We now consider the general case (1.1)-(1.4). The local (in time) existence and uniqueness of a classical solution is proved in $[3]$ :

Proposition $1.1([3])$. Let the assumptions (1.5) $-(1.8)$ be in force. Then

(i) there exists a $T>0$ such that the classical solution exists and is unique in the interval $[0, T)$, where $T$ depends on the $C^{1+\alpha}$ norm of the initial data $u_{0}$;

(ii) if $\xi_{1}(t)+\xi_{2}(t) \geq \delta>0$ for $0<t<T,\|u(\cdot, t)\|_{C^{1+\alpha}\left[-\xi_{1}(t), \xi_{2}(t)\right]} \leq C$ for $0<t<T$, and the $\operatorname{limit}_{\lim } \rightarrow T-0 \xi_{i}(t):=\xi_{i}(T)(i=1,2)$ exists, then there exists a $T^{\prime}>0$ such that the classical solution obtained in (i) can be uniquely extended to the interval $\left[0, T+T^{\prime}\right)$; 
(iii) furthermore, for any $c_{0}>0$, the solution obtained in (i) satisfies $\left(u, \xi_{1}, \xi_{2}\right) \in$ $C^{2+\alpha, 1+\alpha / 2}\left\{(x, t) ;-\xi_{1}(t) \leq x \leq \xi_{2}(t), c_{0} \leq t<T\right\} \times C^{1+\alpha / 2}\left[c_{0}, T\right) \times C^{1+\alpha / 2}\left[c_{0}, T\right)$.

The regularity does not extend to $t=0$, because only $C^{1+\alpha}$ initial data is assumed in [3]. If we assume initial data to be in the class $C^{2+\alpha}$, then the argument in [3] shows that the regularity also extends to $t=0$.

Notice that if $\xi_{i}^{\prime}(t)(i=1,2)$ are bounded on $[0, T)$, then $\lim _{t \rightarrow T-0} \xi_{i}(t)=\xi_{i}(T)$ exists. It is also clear that $W^{2, \infty}$ estimates imply $C^{1+\alpha}$ estimates.

In view of Proposition 1.1 and the discussion above, the following a priori estimates

$$
\begin{aligned}
& \xi_{1}(t)+\xi_{2}(t)>\delta \text { for } t \in[0, T), \quad(\delta>0), \\
& \left|\xi_{1}^{\prime}(t)\right|+\left|\xi_{2}^{\prime}(t)\right| \leq C \text { for } t \in\left[c_{0}, T\right), \\
& \|u(\cdot, t)\|_{W^{2, \infty}\left[-\xi_{1}(t), \xi_{2}(t)\right]} \leq C \text { for } t \in\left[c_{0}, T\right)
\end{aligned}
$$

will be sufficient for us to derive the existence and uniqueness of a classical solution for the system (1.1)-(1.4) in the interval $[0, T)$ and extend the solution to $\left[0, T+T^{\prime}\right)$ for some $T^{\prime}>0$.

Set $\gamma_{1}=\tan \alpha_{1}$ and $\gamma_{2}=\tan \alpha_{2}$. If $\xi_{1}(t)>0$ and $\xi_{2}(t)>0$ for $t \in[0, \tau]$, then by the maximum principle $u(x, t)>0$ for $t \in[0, \tau]$; in this case, we can apply the maximum principle in the regions $\left\{(x, t) ;-\xi_{1}(t)<x<0\right\}$ and $\left\{(x, t) ; 0<x<\xi_{2}(t)\right\}$ respectively to obtain

$$
u(x, t) \geq \max \left[\left(\tan \beta_{2}\right) x,-\left(\tan \beta_{1}\right) x\right], t \in[0, \tau] .
$$

This is shown in Figure 1. Clearly, the area $D(t)$ of the region enclosed by the interface and the two given straight lines is given by (see Figure 1):

$$
D(t)=\int_{-\xi_{1}(t)}^{\xi_{2}(t)} u(y, t) d y-\frac{1}{2} \xi_{1}^{2}(t) \tan \beta_{1}-\frac{1}{2} \xi_{2}^{2}(t) \tan \beta_{2} .
$$

A simple computation shows that, for $t>0$ (notice that $\xi_{i}(t)(i=1,2)$ is continuously differentiable for $t>0$, by Proposition 1.1),

$$
D^{\prime}(t)=a\left(\gamma_{2}\right)-a\left(\gamma_{1}\right)
$$

thus the area $D(t)$ is expanding in the case $\gamma_{1}<\gamma_{2}$, preserved in the case $\gamma_{1}=\gamma_{2}$, and shrinking in the case $\gamma_{1}>\gamma_{2}$.

In the case $\gamma_{1}<\gamma_{2}$, the area is expanding. In this area-expanding case, it is proved in 3 ] that

(i) there exists a unique solution to the system (1.1)-(1.4) for all $0<t<\infty$;

(ii) under the assumption that $\beta_{1}, \beta_{2} \in[0, \pi / 2),-\beta_{1}<\alpha_{1}<\pi / 2$ and $\alpha_{1}<\alpha_{2}<\beta_{2}$, there exists a unique forward self-similar solution to the system (1.1)-(1.3) of the form

$$
u(x, t)=\sqrt{2(t+1)} \varphi\left(\frac{x}{\sqrt{2(t+1)}}\right) ;
$$

(iii) in this expanding case, with appropriate assumptions on the data, the solution of (1.1)-(1.4) will converge to the forward self-similar solution; this is done in [3] through explicit and delicate comparison. 
If $a(s) \equiv s$, then we have the heat equation. In this case, various properties of selfsimilar solutions are discussed in [9]. In particular,

(i) the exact forward self-similar solutions to the system (1.1)-(1.3) of the form (1.15) are studied in the expanding case;

(ii) the exact backward self-similar solutions to the system (1.1)-(1.3) of the form

$$
u(x, t)=\sqrt{2(T-t)} \varphi\left(\frac{x}{\sqrt{2(T-t)}}\right)
$$

are studied in the shrinking case. For a certain range of data, the uniqueness of the backward self-similar solutions is not valid.

The question of asymptotic behavior of the solution to the system (1.1)-(1.4) in the area-preserving and area-shrinking cases is not studied, even for the case of the heat equation $(a(s) \equiv s)$. The goal of this paper is to study these two cases. We begin with the following global existence of the classical solution in the expanding and preserving cases. Note that the global existence for the expanding case has been established in [3]; however, our global existence proof covers both the area-preserving and area-expanding cases.

Theorem 1.2. Consider the expanding and preserving cases $\gamma_{1} \leq \gamma_{2}$. Let the assumptions (1.5) -(1.8) be in force. Assume also that $u_{0} \in C^{1+\alpha}$ for some $\alpha>0$. Then there exists a unique classical solution for $0<t<\infty$.

In the case $\gamma_{1}=\gamma_{2}$, the area is preserved. We have the following result for the asymptotic behavior (the result is new even for the heat equation case).

Theorem 1.3. Consider the area-preserving case $\gamma_{1}=\gamma_{2}$. Let the assumptions (1.5)(1.8) be in force. Then there exists $d_{1}>0$, determined uniquely by the initial data, such that

$$
u(x, t) \rightarrow \gamma x+d_{1}
$$

uniformly in $x$ as $t \rightarrow \infty$.

In the shrinking case when $\gamma_{1}>\gamma_{2}$, the area should vanish in finite time. We shall establish the asymptotic behavior in this case (Theorems 1.4 and 1.5). The result is new even for the heat equation case.

Theorem 1.4. Consider the area-shrinking cases $\gamma_{1}>\gamma_{2}$. Let the assumptions (1.5)(1.8) be in force. We further assume

$$
-\beta_{1} \leq \alpha_{2}<\alpha_{1} \leq \beta_{2} .
$$

Then

(i) there exists a unique classical solution $\left(u(x, t), \xi_{1}(t), \xi_{2}(t)\right)$ to the system (1.1)-(1.4) for $0 \leq t<T$ with $T$ given by

$$
T=D(0) /\left[a\left(\gamma_{1}\right)-a\left(\gamma_{2}\right)\right],
$$

where

$$
D(0)=\int_{-\xi_{10}}^{\xi_{20}} u_{0}(x) d x-\frac{1}{2} \xi_{10}^{2} \tan \beta_{1}-\frac{1}{2} \xi_{20}^{2} \tan \beta_{1} .
$$


(ii) $u \in C^{2+\alpha, 1+\alpha / 2}\left(\Omega_{T}\right) \cap C^{0+1,1 / 2}\left(\bar{\Omega}_{T}\right)$ and $\xi_{1}, \xi_{2} \in C^{1+\alpha / 2}(0, T) \cap C^{1 / 2}[0, T]$, where $\Omega_{T}=\left\{(x, t) ;-\xi_{1}(t) \leq x \leq \xi_{2}(t), 0<t<T\right\}$.

(iii) $\lim _{t \rightarrow T-0}\|u(\cdot, t)\|_{L^{\infty}}=0$ and $\xi_{1}(T)=\xi_{2}(T)=0$.

To study the asymptotic behavior at the vanishing time $T$, we make a change of variables and define $(v(y, s), p(s), q(s))$ by

$$
\begin{aligned}
& u(x, t)=\sqrt{2(T-t)} v(y, s), \quad y=\frac{x}{\sqrt{2(T-t)}}, T-t=e^{-2 s}, \\
& \xi_{1}(t)=\sqrt{2(T-t)} p(s), \quad \xi_{2}(t)=\sqrt{2(T-t)} q(s) .
\end{aligned}
$$

Then

$$
\begin{aligned}
& v_{s}=\left[a\left(v_{y}\right)\right]_{y}-y v_{y}+v,-p(s)<y<q(s), s>s_{0}:=-\ln (T) / 2, \\
& v_{y}(-p(s), s)=\gamma_{1}, v_{y}(q(s), s)=\gamma_{2}, s>s_{0}, \\
& v(-p(s), s)=p(s) \tan \beta_{1}, v(q(s), s)=q(s) \tan \beta_{2}, s>s_{0} .
\end{aligned}
$$

The steady-state solutions $(\varphi, p, q)$ of (1.19) $-(1.21)$ are given by

$$
\begin{aligned}
& {\left[a\left(\varphi^{\prime}\right)\right]^{\prime}-y \varphi^{\prime}+\varphi=0, \quad-p<y<q, \quad p>0, \quad q>0,} \\
& \varphi^{\prime}(-p)=\gamma_{1}, \varphi^{\prime}(q)=\gamma_{2}, \\
& \varphi(-p)=p \tan \beta_{1}, \quad \varphi(q)=q \tan \beta_{2} .
\end{aligned}
$$

Theorem 1.5. Let the assumptions (1.5)-(1.8) be in force. We further assume

$$
-\beta_{1}<\alpha_{2}<\alpha_{1}<\beta_{2} \text {. }
$$

Let $T$ be given as in (1.18) and $(v, p, q)$ be defined as above. Then

(i) for any initial data in $C^{1+\alpha}$, the corresponding $\omega$-limit set is not empty;

(ii) any $\omega$-limit is a solution to (1.22)-(1.24);

(ii) if we further assume $a(s)$ to be analytic, then $v(y, s)$ will converge to one of the $\omega$-limits as $s \rightarrow \infty$.

REMARK 1.1. The backward self-similar solutions in the case $a(s) \equiv s$ were extensively studied in [9], where existence and nonuniqueness as well as the properties of solutions were discussed. In the case of general $a(s)$, Theorem 1.5 establishes the existence of such a backward self-similar solution under the additional assumption (1.25).

This paper is organized as follows. In section 2, we derive some a priori estimates for solutions of (FBP). Also, we give a proof of the global existence of solutions of (FBP) in the area-preserving and area-expanding cases (Theorem 1.2). We then give a proof for the convergence of the solution to a line in the area-preserving case in section 3 (Theorem 1.3). In section 4, we turn to the shrinking case and give some preliminary results, where we also prove the existence result as well as the finite-time vanishing result (Theorem 1.4). Finally, we study in section 5 the asymptotic behavior of the solution of (FBP) in the shrinking case and prove Theorem 1.5. The convergence result in section 5 depends also on the properties of the backward self-similar solutions derived in section 6 . 
2. Some a priori estimates. In this section we derive some a priori estimates for solutions of (FBP). For the estimates in this section, we shall always assume (1.5)-(1.8). Note that under these assumptions, the free boundaries $x=\xi_{i}(t)(i=1,2)$ and their first-order derivatives are Hölder continuous for $t>0$ (Proposition 1.1). These regularity properties of the free boundary should be used throughout this paper.

We first get the $L^{\infty}$ bounds on $u_{x}$, which is a simple application of the comparison principle. We assume that the classical solution exists on $[0, \tau)$, for some $0<\tau \leq+\infty$.

LEMMA 2.1.

$$
\left|u_{x}(x, t)\right| \leq C_{1} \quad \text { for } \quad 0<t<\tau,
$$

where

$$
C_{1}=\max \left(\left|\gamma_{1}\right|,\left|\gamma_{2}\right|,\left\|\left(u_{0}\right)_{x}\right\|_{L^{\infty}}\right) .
$$

Note that Lemma 2.1 implies that $a^{\prime}\left(u_{x}(x, t)\right)$ is uniformly bounded from above and bounded from below by a positive constant. Thus the equation (1.1) becomes uniformly parabolic.

We next derive half-sided estimates for $u_{x x}$.

Lemma 2.2. For any $c_{0}>0$, there exists a constant $C$ such that

$$
u_{x x} \leq C \text { for }-\xi_{1}(t)<x<\xi_{2}(t), c_{0}<t<\tau .
$$

Proof. Differentiating boundary conditions (1.2), (1.3) at $x=\xi_{2}(t)$ in $t$, we obtain

$$
u_{x x}\left(\xi_{2}(t), t\right) \xi_{2}^{\prime}(t)+u_{x t}\left(\xi_{2}(t), t\right)=0, \quad u_{x}\left(\xi_{2}(t), t\right) \xi_{2}^{\prime}(t)+u_{t}\left(\xi_{2}(t), t\right)=\xi_{2}^{\prime}(t) \tan \beta_{2} .
$$

Using the equation (1.1) and the boundary condition (1.2), we now get

$$
\begin{aligned}
u_{t x}\left(\xi_{2}(t), t\right) & =-u_{x x}\left(\xi_{2}(t), t\right) \xi_{2}^{\prime}(t) \\
& =-\frac{u_{t}\left(\xi_{2}(t), t\right)}{a^{\prime}\left(u_{x}\left(\xi_{2}(t), t\right)\right)} \xi_{2}^{\prime}(t) \\
& =-\frac{u_{t}\left(\xi_{2}(t), t\right)}{a^{\prime}\left(u_{x}\left(\xi_{2}(t), t\right)\right)}\left(\frac{u_{t}\left(\xi_{2}(t), t\right)}{\tan \beta_{2}-u_{x}\left(\xi_{2}(t), t\right)}\right) \\
& =-\frac{1}{\left(\tan \beta_{2}-\gamma_{2}\right) a^{\prime}\left(\gamma_{2}\right)} u_{t}^{2}\left(\xi_{2}(t), t\right) \leq 0 .
\end{aligned}
$$

Similarly,

$$
-u_{t x}\left(-\xi_{1}(t), t\right)=-\frac{1}{\left(\tan \beta_{1}+\gamma_{1}\right) a^{\prime}\left(\gamma_{1}\right)} u_{t}^{2}\left(\xi_{1}(t), t\right) \leq 0 .
$$

It is clear that $u_{t}$ satisfies

$$
u_{t t}=a^{\prime}\left(u_{x}\right) u_{t x x}+a^{\prime \prime}\left(u_{x}\right) u_{t x} .
$$

It is proved in [3] that $u_{x x}(x, t)$ is bounded for $t=c_{0}$ for small $c_{0}>0$, provided we assume $u_{0} \in C^{1+\alpha}$ for some $\alpha \in(0,1)$. It follows that $u_{t}=a^{\prime}\left(u_{x}\right) u_{x x}$ is bounded for $t=c_{0}$. Thus we can apply the maximum principle to $u_{t}$ to conclude that $u_{t}$ is bounded from above. This implies that $u_{x x}=u_{t} / a^{\prime}\left(u_{x}\right)$ is bounded from above.

REMARK 2.1. If $u_{0} \in C^{2}$ and satisfies the second-order compatibility condition at the boundary, then we can take $c_{0}=0$ in the above lemma.

We next derive the lower bound for $u_{x x}$. 
LEMma 2.3. If

$$
u(0, t) \geq m \text { for } 0<t<\tau,
$$

for some $m>0$, then for any $c_{0}>0$, there exists $C$ independent of $m$ such that

$$
u_{x x} \geq-\frac{C}{m} \text { for } \quad-\xi_{1}(t)<x<\xi_{2}(t), c_{0}<t<\tau .
$$

Proof. By changing the value of $a(p)$ outside $|p|>C_{1}$ (where $C_{1}$ is from (2.2) in Lemma 2.1) if necessary, we may assume without loss of generality that $l<a^{\prime}(p)<1 / l$ for all $p \in(-\infty, \infty)$ for some $l>0$ and $a \in C^{2}$. This implies that the inverse function of $a^{-1}(\cdot)$ is well defined on $(-\infty, \infty)$.

We choose $w$ such that

$$
a(w)=\frac{C}{m}\left(u-x \tan \beta_{2}\right)+a\left(\gamma_{2}\right) .
$$

If we take $C$ such that $C+a\left(\gamma_{2}\right) \geq a\left(C_{1}\right)$, then $u_{x}(0, t) \leq w(0, t), u_{x}\left(\xi_{2}(t), t\right)=\gamma_{2}=$ $w\left(\xi_{2}(t), t\right)$ for $0<t<\tau$. Since $u_{x x}$ is bounded for $t=c_{0}$ and $\tan \beta_{2}-u_{x}\left(\xi_{2}\left(c_{0}\right), c_{0}\right)=$ $\tan \beta_{2}-\gamma_{2}>0$, there exists $\varepsilon>0$ such that $\tan \beta_{2}-u_{x}\left(x, c_{0}\right)>\left(\tan \beta_{2}-\gamma_{2}\right) / 2$ for $x \in\left[\xi_{2}\left(c_{0}\right)-\varepsilon, \xi_{2}\left(c_{0}\right)\right]$. Using the bounds on $u_{x x}$ and $u_{x}$, we can choose $C$ large enough so that

$$
\begin{aligned}
u_{x x}\left(x, c_{0}\right) & >-\frac{C l}{2 m}\left(\tan \beta_{2}-\gamma_{2}\right) \\
& \geq-\frac{C}{m a^{\prime}(w)}\left(\tan \beta_{2}-u_{x}\left(x, c_{0}\right)\right) \\
& =w_{x}\left(x, c_{0}\right) \text { for } x \in\left[\xi_{2}\left(c_{0}\right)-\varepsilon, \xi_{2}\left(c_{0}\right)\right] .
\end{aligned}
$$

Integrating this inequality over the interval $\left[x, \xi_{2}\left(c_{0}\right)\right]$, using also the boundary condition $u_{x}\left(\xi_{2}\left(c_{0}\right), c_{0}\right)=w\left(\xi_{2}\left(c_{0}\right), c_{0}\right)=\gamma_{2}$, we find that

$$
u_{x}\left(x, c_{0}\right) \leq w\left(x, c_{0}\right) \text { for } \xi_{2}\left(c_{0}\right)-\varepsilon \leq x \leq \xi_{2}\left(c_{0}\right) .
$$

Since $u-x \tan \beta_{2}$ is positive on the interval $x \in\left[0, \xi_{2}\left(c_{0}\right)-\varepsilon\right]$, it is clear that the above inequality is valid for $0<x<\xi_{2}\left(c_{0}\right)-\varepsilon$ if we choose the constant $C$ in the definition of $w$ to be large enough.

Now that the constant $C$ in $w$ is fixed. We can finally define the uniformly parabolic operator $\mathcal{L}$ (in the definition we fix on $u_{x}, u_{x x}$ and $w$ ) by

$$
\mathcal{L}[\varphi]=\varphi_{t}-[a(\varphi)]_{x x}-\frac{C}{m a^{\prime}(w)} u_{x x}\left[a^{\prime}\left(u_{x}\right)-a^{\prime}(\varphi)\right] .
$$

Clearly

$$
\mathcal{L}\left[u_{x}\right]=u_{x t}-\left[a\left(u_{x}\right)\right]_{x x}-\frac{C}{m a^{\prime}(w)} u_{x x}\left[a^{\prime}\left(u_{x}\right)-a^{\prime}\left(u_{x}\right)\right]=0
$$


and

$$
\begin{aligned}
\mathcal{L}[w] & =w_{t}-[a(w)]_{x x}-\frac{C}{m a^{\prime}(w)} u_{x x}\left[a^{\prime}\left(u_{x}\right)-a^{\prime}(w)\right] \\
& =\frac{C}{m}\left(\frac{1}{a^{\prime}(w)} u_{t}-u_{x x}\right)-\frac{C}{m a^{\prime}(w)} u_{x x}\left[a^{\prime}\left(u_{x}\right)-a^{\prime}(w)\right] \\
& =\frac{C}{m}\left(\frac{1}{a^{\prime}(w)} a^{\prime}\left(u_{x}\right) u_{x x}-u_{x x}\right)-\frac{C}{m a^{\prime}(w)} u_{x x}\left[a^{\prime}\left(u_{x}\right)-a^{\prime}(w)\right] \\
& =0
\end{aligned}
$$

Thus by comparison

$$
u_{x}<w \text { for } 0<x<\xi_{2}(t), \quad c_{0}<t<\tau .
$$

Thus

$$
u_{x x}\left(\xi_{2}(t), t\right) \geq w_{x}\left(\xi_{2}(t), t\right) \geq-\frac{C}{m l}\left(\tan \beta_{2}-\gamma_{2}\right) \quad \text { for } \quad c_{0}<t<\tau .
$$

One can similarly show that

$$
u_{x}>a^{-1}\left(-\frac{C}{m}\left(u+x \tan \beta_{1}\right)+a\left(\gamma_{1}\right)\right) \quad \text { for } \quad-\xi_{1}(t)<x<0, \quad c_{0}<t<\tau
$$

and obtain

$$
u_{x x}\left(-\xi_{1}(t), t\right) \geq-\frac{C}{m l}\left(\gamma_{1}+\tan \beta_{1}\right) \quad \text { for } \quad c_{0}<t<\tau .
$$

These estimates imply that (by using the equation)

$$
u_{t}(x, t) \geq-\frac{C^{*}}{m} \text { for } x=-\xi_{1}(t), x=\xi_{2}(t), c_{0}<t<\tau .
$$

Differentiating the equation (1.1) in $t$ and applying the maximum principle to $u_{t}$, we obtain

$$
u_{t} \geq-\frac{C^{*}}{m} \text { for }-\xi_{1}(t)<x<\xi_{2}(t), c_{0}<t<\tau .
$$

Using the equation (1.1) again, we conclude the lemma.

These estimates imply the classical global existence in the area-preserving and areaexpanding cases as claimed in Theorem 1.2

Proof of Theorem 1.2. We can choose any $\gamma \in\left[\gamma_{1}, \gamma_{2}\right]$ and $d>0$ such that $u_{0}(x) \geq$ $\gamma x+d$ for $x \in\left[-\xi_{1}(0), \xi_{2}(0)\right]$. Then by comparison, we immediately get

$$
u(x, t) \geq \gamma x+d \text { for } \quad-\xi_{1}(t)<x<\xi_{2}(t) .
$$

By Lemma 2.1 $u_{x}$ is uniformly bounded by $C_{1}$. By (2.8), $u(0, t) \geq d$. Thus if we choose $m=d$ in Lemma 2.3, then $u_{x x}$ is also uniformly bounded for $t>c_{0}$, which in turn implies that $u_{t}$ is bounded for $t>c_{0}$ by using the equation (1.1). The estimate (2.8) also implies

$$
\min \left[\xi_{1}(t), \xi_{2}(t)\right] \geq \delta
$$

for some $\delta>0$. From (2.4), (1.2) and the uniform bound on $u_{t}$, we conclude that

$$
\xi_{2}^{\prime}(t)=\frac{u_{t}\left(\xi_{2}(t), t\right)}{\tan \beta_{2}-\gamma_{2}}
$$

is uniformly bounded for $t>t_{0}$. Similarly, $\xi_{2}^{\prime}(t)$ is also bounded for $t>c_{0}$. Therefore $\xi_{i}(t)(i=1,2)$ are bounded by $C t+C$ and $u(x, t)$ is therefore also bounded by $C t+C$. 
Using these bounds in Proposition 1.1, we find that the solution can be extended to all $t$.

REMARK 2.2. We proved that for the case $\gamma_{1} \leq \gamma_{2}$, the solution is global, and

$$
\sup _{c_{0}<t<\infty}\left\{\|u(\cdot, t)\|_{W^{2, \infty}\left[-\xi_{1}(t), \xi_{2}(t)\right]}+\left\|u_{t}(\cdot, t)\right\|_{L^{\infty}\left[-\xi_{1}(t), \xi_{2}(t)\right]}+\left|\xi_{1}^{\prime}(t)\right|+\left|\xi_{2}^{\prime}(t)\right|\right\} \leq C .
$$

This estimate is useful in the asymptotic study for the area-preserving case.

3. Asymptotic limit for the area-preserving case. For the case $\gamma_{1} \geq \gamma_{2}$, we can choose any $\gamma \in\left[\gamma_{2}, \gamma_{1}\right]$ and $D>0$ such that $u_{0}(x) \leq \gamma x+D$ for $x \in\left[-\xi_{1}(0), \xi_{2}(0)\right]$. Then by comparison, we immediately get

$$
u(x, t) \leq \gamma x+D \text { for }-\xi_{1}(t)<x<\xi_{2}(t) .
$$

Choosing $\gamma=\gamma_{1}$ and $\gamma=\gamma_{2}$ respectively, using also (1.3), we obtain

$$
\xi_{1}(t) \leq \frac{D}{\tan \beta_{1}+\gamma_{1}}, \quad \xi_{2}(t) \leq \frac{D}{\tan \beta_{2}-\gamma_{2}} .
$$

In particular, if $\gamma_{1}=\gamma_{2}:=\gamma$, then we can combine (2.8) and (3.1):

$$
\gamma x+d \leq u(x, t) \leq \gamma x+D \text { for }-\xi_{1}(t)<x<\xi_{2}(t), 0<t<\infty .
$$

By (2.11), we also have uniform bounds on $u_{x}, u_{x x}, \xi_{i}^{\prime}(t)(i=1,2)$ for $t \in\left[c_{0}, \infty\right)$.

Proof of Theorem 1.3, For

$$
A(s):=\int_{0}^{s} a(y) d y
$$

and

$$
J(t)=\int_{-\xi_{1}(t)}^{\xi_{2}(t)} A\left(u_{x}(x, t)\right) d x
$$

we have (using the boundary conditions)

$$
\begin{aligned}
J^{\prime}(t)= & \int_{-\xi_{1}(t)}^{\xi_{2}(t)} a\left(u_{x}\right) u_{x t} d x+A(\gamma) \xi_{2}^{\prime}(t)+A(\gamma) \xi_{1}^{\prime}(t) \\
= & -\int_{-\xi_{1}(t)}^{\xi_{2}(t)}\left[a\left(u_{x}\right)\right]_{x} u_{t} d x+\left.a\left(u_{x}\right) u_{t}\right|_{-\xi_{1}(t)} ^{\xi_{2}(t)}+A(\gamma) \xi_{2}^{\prime}(t)+A(\gamma) \xi_{1}^{\prime}(t) \\
= & -\int_{-\xi_{1}(t)}^{\xi_{2}(t)} u_{t}^{2} d x+\left[a(\gamma)\left(\tan \beta_{2}-\gamma\right)+A(\gamma)\right] \xi_{2}^{\prime}(t) \\
& +\left[-a(\gamma)\left(\tan \beta_{1}+\gamma\right)+A(\gamma)\right] \xi_{1}^{\prime}(t) .
\end{aligned}
$$

Thus

$$
J(t)+\int_{c_{0}}^{t} \int_{-\xi_{1}(t)}^{\xi_{2}(t)} u_{t}^{2} d x \leq J\left(c_{0}\right)+C\left(\left\|\xi_{1}\right\|_{L^{\infty}\left[c_{0}, \infty\right)}+\left\|\xi_{2}\right\|_{L^{\infty}\left[c_{0}, \infty\right)}\right) .
$$

The right-hand side of the above equality is uniformly bounded. Thus

$$
\int_{c_{0}}^{\infty} \int_{-\xi_{1}(t)}^{\xi_{2}(t)} u_{t}^{2} d x<\infty
$$

This estimate, together with the compactness of the solution as $t \rightarrow \infty$ (subsequence of $u\left(x, t+t_{j}\right)$ converges in $C^{1+\alpha}$ in $x$ up to the boundary and $C^{2}$ in the interior, $\xi_{i}\left(t_{j}\right) \rightarrow \xi_{i \infty}$ $(i=1,2))$, we immediately obtain the convergence on the subsequence. By following the 
standard argument as in [7, we find that the subsequential limit function must be a solution to the steady state equation $\left[a\left(u_{x}\right)\right]_{x}=0$ with the boundary conditions (1.2), (1.3). It is clear that the only solutions to the steady state are lines of the type $\gamma x+d_{1}$ for some $d_{1}$.

With the $D(t)$ defined in (1.6), it is clear that $D^{\prime}(t)=0$ in the area-preserving case. In particular, $D(t) \equiv D(0)$ for all $t>0$. If we pass to the limit along the convergent subsequence, we find

$$
\int_{-\xi_{1 \infty}}^{\xi_{2 \infty}}\left(\gamma x+d_{1}\right) d x-\frac{1}{2} \xi_{1 \infty}^{2} \tan \beta_{1}-\frac{1}{2} \xi_{2 \infty}^{2} \tan \beta_{2}=D(0) .
$$

From (1.3), we also have

$$
-\gamma \xi_{1 \infty}+d_{1}=\xi_{1 \infty} \tan \beta_{1}, \quad \gamma \xi_{2 \infty}+d_{1}=\xi_{2 \infty} \tan \beta_{2} .
$$

It is clear that (3.5) and (3.6) uniquely determine $d_{1}, \xi_{1 \infty}$ and $\xi_{2 \infty}$. As in [7, the uniqueness of the limit $\left(d_{1}, \xi_{1 \infty}, \xi_{2 \infty}\right)$ implies that $u(x, t), \xi_{i}(t)(i=1,2)$ all converge uniformly as $t \rightarrow \infty$.

REMARK 3.1. Let $b=\lim _{t \rightarrow \infty}\left[\xi_{1}(t)+\xi_{2}(t)\right]$. The convergence theorem of the standard parabolic theory gives the exponential convergence rate up to the first eigenvalue (see [5] ; the convergence rate for the linearized problem, the uniform convergence of the nonlinear problem, together with the convergence rate for the linearized problem, imply the convergence rate for the nonlinear problem). More precisely, for any $\lambda \in\left(0, a^{\prime}(\gamma) \pi^{2} / b^{2}\right)$, $\lim _{t \rightarrow \infty} e^{\lambda t}\left|u(x, t)-\gamma x-d_{1}\right|=0$.

4. Preliminary for the shrinking case. The assumption $\gamma_{1}>\gamma_{2}$ means that there is a negative total heat flux through the boundary and therefore $u$ should decrease overall.

Notice that the estimates (3.1) and (3.2) were proved for both the area-preserving and area-shrinking cases. These bounds are valid as long as the solution exists:

$$
u(x, t) \leq \gamma x+D, \quad \xi_{1}(t) \leq C, \quad \xi_{2}(t) \leq C .
$$

In this section we assume that $[0, T)$ is the maximal existence interval for a classical solution. In the Lemmas 4.14.4 in this section, we only need to assume that the initial data $u_{0}$ is $C^{1+\alpha}$ and we do not need the condition (1.17).

We first prove the Hölder estimates for $u(x, t)$ in the $t$ direction. We extend $u(x, t)$ to all $x \in(-\infty, \infty)$ by defining

$$
u(x, t)=\left\{\begin{array}{l}
-x \tan \beta_{1} \text { for } x<-\xi_{1}(t) \\
x \tan \beta_{2} \text { for } x>\xi_{2}(t)
\end{array}\right.
$$

Lemma 4.1. The extended function $u(x, t)$ satisfies, for any $t_{1}, t_{2} \in[0, T)$,

$$
\left|u\left(x, t_{1}\right)-u\left(x, t_{2}\right)\right| \leq C\left|t_{1}-t_{2}\right|^{1 / 2} .
$$

Proof. The extended function satisfies the equation (1.1) both inside and outside the region $\left\{(x, t), \quad x \in\left(-\xi_{1}(t), \xi_{2}(t)\right)\right\}$. There is a jump in the first-order $x$-derivative 
across the boundary, but $\left|u_{x}\right|$ is uniformly bounded by $\max \left\{C_{1}, \tan \beta_{1}, \tan \beta_{2}\right\}$ for all $x \in(-\infty, \infty)$, and $u$ satisfies in the distribution sense the equation

$u_{t}=\frac{\partial}{\partial x}\left[a\left(u_{x}\right)+\left\{a\left(\gamma_{2}\right)-a\left(\tan \beta_{2}\right)\right\} H\left(x-\xi_{2}(t)\right)-\left\{a\left(\gamma_{1}\right)-a\left(-\tan \beta_{1}\right)\right\} H\left(x+\xi_{1}(t)\right)\right]$, where $H(t)$ is the Heaviside function. It follows that, for any $x_{1}, x_{2}$ and $t_{1}<t_{2}$,

$$
\left|\int_{x_{1}}^{x_{2}}\left(u\left(x, t_{1}\right)-u\left(x, t_{2}\right)\right) d x\right| \leq \int_{t_{1}}^{t_{2}}\left|\int_{x_{1}}^{x_{2}} u_{t}(x, \tau) d x\right| d \tau \leq C\left|t_{1}-t_{2}\right| .
$$

For any given $x^{*}$ and $t_{1}, t_{2}$, we choose $x_{1}, x_{2}$ such that $x_{1}<x^{*}<x_{2}$ and $\left|x_{1}-x_{2}\right|=$ $\left|t_{1}-t_{2}\right|^{1 / 2}$. Then

$$
\begin{aligned}
& \left|u\left(x^{*}, t_{1}\right)-u\left(x^{*}, t_{2}\right)\right| \\
& \leq \frac{1}{\left|x_{1}-x_{2}\right|}\left|\int_{x_{1}}^{x_{2}}\left(u\left(x, t_{1}\right)-u\left(x, t_{2}\right)\right) d x\right|+|| u_{x} \|_{L^{\infty}}\left|x_{1}-x_{2}\right| \\
& \leq C\left|t_{1}-t_{2}\right|^{1 / 2} .
\end{aligned}
$$

This completes the proof.

If we replace the positiveness assumption on $u(0, t)$ by certain assumptions on $u\left(x_{0}, t\right)$, we can repeat the proof of Lemma 2.3 to conclude :

Lemma 4.2. If

$$
u\left(x_{0}, t\right)-\max \left[\left(\tan \beta_{2}\right) x_{0},-\left(\tan \beta_{1}\right) x_{0}\right]>m \text { for } 0<t<\tau,
$$

for some $m>0$, then for any $c_{0}>0$, there exists $C$ independent of $m$ such that

$$
u_{x x} \geq-\frac{C}{m} \text { for }-\xi_{1}(t)<x<\xi_{2}(t), c_{0}<t<\tau .
$$

For a positive solution, we have

Lemma 4.3. If $u(x, t)>0$ for $t \in[0, \tau]$, then

$$
u(x, t)>\max \left[\left(\tan \beta_{2}\right) x,-\left(\tan \beta_{1}\right) x\right], x \in\left(-\xi_{1}(t), \xi_{2}(t)\right), \xi_{1}(t)>0, \xi_{2}(t)>0
$$

for $t \in[0, \tau]$.

Proof. Using the boundary condition, we can compare the solution with the functions $\left(\tan \beta_{2}\right) x$ and $-\left(\tan \beta_{1}\right) x$ respectively to conclude the result.

We now prove that the positive solution must vanish at $t=T$.

Lemma 4.4. Suppose that $[0, T)$ is the maximal existence interval for the classical solution and $T<\infty$. If $u(x, t)$ remains positive on $\left\{(x, t),-\xi_{1}(t)<x<\xi_{2}(t), 0<t<T\right\}$, then

$$
\lim _{t \rightarrow T-} \xi_{1}(t)=0, \quad \lim _{t \rightarrow T-} \xi_{2}(t)=0 .
$$

Proof. Since $u_{t}$ is bounded above by Lemma 2.2. $\xi_{i}^{\prime}(t) \leq C(i=1,2)$ (cf. (2.10) $)$; the function $\xi_{i}(t)-C t$ is therefore monotonically decreasing and the limit $\lim _{t \rightarrow T-}\left(\xi_{i}(t)-C t\right)$ exists. Therefore the limit $\lim _{t \rightarrow T-} \xi_{i}(t)$ exists, and we define it to be $\xi_{i}(T)$. With this definition, it is clear that $\xi_{i}(t)$ is continuous on $[0, T]$. If $\xi_{1}(T)+\xi_{2}(T)>0$, then for $x_{0}=\left[\xi_{1}(T)+\xi_{2}(T)\right] / 2$, we have $u\left(x_{0}, T\right)>\max \left[\left(\tan \beta_{2}\right) x_{0},-\left(\tan \beta_{1}\right) x_{0}\right]$, by Lemma 4.3 
and the strong maximum principle. Now we can apply Lemma 4.2 to conclude that $u_{x x}$ remains bounded near $t=T$ and therefore the classical solution can be further extended beyond $t=T$. This contradicts our assumption that $[0, T)$ is the maximal existence interval. Thus we must have $\xi_{1}(T)+\xi_{2}(T)=0$ and the lemma is proved.

Next we give a sufficient condition for the solution $u$ to remain positive.

If we have the additional assumption (1.17), then we claim that $u$ is always positive as follows.

Lemma 4.5. Suppose that $[0, T)$ is the maximal existence interval for the classical solution and $T<\infty$. Under the additional assumption (1.17), we have $u(x, t)>0$ for $0 \leq t<T$. In particular, Lemma 4.4 implies that $\xi_{1}(T)=\xi_{2}(T)=0$ and $u(0, T)=0$.

Proof. Let $\left[0, T^{*}\right)$ be the maximal interval on which $u(x, t)$ remains positive. Then both $\xi_{1}$ and $\xi_{2}$ are positive on this interval. We claim that $T^{*}=T$. In fact, if $T^{*}<T$, then $u(x, t)$ is $C^{2}$ for $t \in\left[0, T^{*}\right]$ and $\xi_{1}\left(T^{*}\right)+\xi_{2}\left(T^{*}\right)>0$. Lemma 4.3 implies that $u\left(x, T^{*}\right)$ is positive for $x \neq 0$. Therefore we must have $u\left(0, T^{*}\right)=0$. If $-\xi_{1}\left(T^{*}\right)<0<\xi_{2}\left(T^{*}\right)$, then $u(x, t)$ reaches the minimum at an interior point $\left(0, T^{*}\right)$ and therefore $u \equiv 0$ and we get a contradiction. If $\xi_{1}\left(T^{*}\right)=0$, then $\xi_{2}\left(T^{*}\right)>0$. Using Lemma 4.3 and applying the strong maximum principle to $u-\left(\tan \beta_{2}\right) x$, we obtain $u_{x}\left(0, T^{*}\right)-\tan \beta_{2}>0$, i.e., $\tan \alpha_{1}>\tan \beta_{2}$. This contradicts (1.17). We can similarly get a contradiction if $\xi_{2}\left(T^{*}\right)=0$. Thus we proved $T^{*}=T$, and $u(x, t)>0$ for $0 \leq t<T$.

We are now ready to prove Theorem 1.4 .

Proof of Theorem 1.4. In view of Lemmas 4.1 4.5, the parts (ii) and (iii) of Theorem 1.4 are already established if we can establish (i), namely, for the maximal existence interval $[0, T), T$ is finite and is given in (1.18). Let us denote the right-hand side of (1.18) to be $T^{*}$, i.e., $T^{*}=D(0) /\left[a\left(\gamma_{1}\right)-a\left(\gamma_{2}\right)\right]$ and we want to show $T=T^{*}$. It is clear from (1.14) that $T^{*}$ is the unique time such that

$$
D(t)>0 \text { for } 0<t<T^{*}, D\left(T^{*}\right)=0 .
$$

By Lemma 4.5, $u(x, t)>0$ on the maximal existence interval $[0, T)$. Then using Lemma 4.3, we find that the $D(t)$ defined in (1.13) satisfies

$$
D(t)>0 \text { for } 0 \leq t<T \text {. }
$$

By Lemma 4.4, $D(T)=0$. By comparing this with (4.4), we conclude that $T=T^{*}$. This finishes the proof.

The rest of this section will be devoted to deriving additional estimates needed for the proof of the asymptotic expansion. If we replace (1.17) with (1.25), we can also derive the estimate on $\xi_{j}(t), j=1,2$.

Lemma 4.6. Suppose that $[0, T)$ is the maximal existence interval for the classical solution, where $T$ is given by (1.18). Under the further assumption (1.25),

$$
0<\xi_{j}(t)<C \sqrt{T-t} \text { for } j=1,2, t<T .
$$

Proof. First, it follows from Lemmas 4.3 and 4.5 that $\xi_{j}(t)>0$ for $j=1,2, t<T$. Integrating the inequality (2.3), we obtain

$$
\gamma_{2}-C\left(\xi_{2}(t)-x\right) \leq u_{x}(x, t) \leq \gamma_{1}+C\left(x+\xi_{1}(t)\right) \quad \text { for } \quad-\xi_{1}(t)<x<\xi_{2}(t), t<T .
$$


Integrating this inequality again, we get

$$
u(x, t) \leq\left\{\begin{array}{l}
u(0, t)+\gamma_{1} x+C\left(\frac{1}{2} x^{2}+x \xi_{1}(t)\right) \text { for } \quad 0<x<\xi_{2}(t), \\
u(0, t)+\gamma_{2} x+C\left(\frac{1}{2} x^{2}-x \xi_{2}(t)\right) \text { for } \quad-\xi_{1}(t)<x<0 .
\end{array}\right.
$$

Thus

$$
\int_{-\xi_{1}(t)}^{\xi_{2}(t)} u(x, t) d x \leq\left[\xi_{1}(t)+\xi_{2}(t)\right] u(0, t)+\frac{\gamma_{1}}{2} \xi_{2}^{2}(t)-\frac{\gamma_{2}}{2} \xi_{1}^{2}(t)+\widetilde{C}\left[\xi_{1}^{3}(t)+\xi_{2}^{3}(t)\right] .
$$

Substituting this estimate into the expression for $D(t)$ in (1.13), we derive

$$
\begin{aligned}
& D(t)+\frac{1}{2}\left(\tan \beta_{1}+\gamma_{2}\right) \xi_{1}^{2}(t)+\frac{1}{2}\left(\tan \beta_{2}-\gamma_{1}\right) \xi_{2}^{2}(t) \\
& \quad \leq\left[\xi_{1}(t)+\xi_{2}(t)\right] u(0, t)+\widetilde{C}\left[\xi_{1}^{3}(t)+\xi_{2}^{3}(t)\right] .
\end{aligned}
$$

Notice that the assumption (1.25) implies that the coefficients $\left(\tan \beta_{1}+\gamma_{2}\right)$ and $\left(\tan \beta_{2}-\right.$ $\left.\gamma_{1}\right)$ are positive. Since $D(t)>0$ for $t<T$, (4.8) implies, for $r(t)=\sqrt{\xi_{1}^{2}(t)+\xi_{2}^{2}(t)}$,

$$
r^{2}(t)\left[1-C^{*} r(t)\right] \leq C r(t) u(0, t) .
$$

Choosing $t$ to be sufficiently close to $T$ such that $1-C^{*} r(t) \leq 1 / 2$, we then obtain

$$
r(t) \leq C u(0, t) \leq C \sqrt{T-t} .
$$

The last inequality was obtained from the Hölder continuity in $t$ for $u(0, t)$ (Lemma 4.1). This completes the proof.

We finally prove

Lemma 4.7. Under the assumptions of Lemma 4.6, there exists $c>0$ such that

$$
u(0, t) \geq c \sqrt{T-t} .
$$

Proof. Using Lemma 4.6 in (4.8), we obtain

$$
\left[a\left(\gamma_{1}\right)-a\left(\gamma_{2}\right)\right](T-t)=D(t) \leq C \sqrt{T-t} u(0, t)+C(T-t)^{3 / 2},
$$

from which the lemma follows.

5. Asymptotic behavior for the shrinking case. In this section we want to study the asymptotic behavior as $t \rightarrow T$. Throughout this section we shall assume that (1.25) is satisfied. Let

$$
\begin{aligned}
& u(x, t)=\sqrt{2(T-t)} v(y, s), \quad y=\frac{x}{\sqrt{2(T-t)}}, \quad T-t=e^{-2 s}, \\
& \xi_{1}(t)=\sqrt{2(T-t)} p(s), \quad \xi_{2}(t)=\sqrt{2(T-t)} q(s) .
\end{aligned}
$$

Then

$$
\begin{aligned}
& v_{s}=\left[a\left(v_{y}\right)\right]_{y}-y v_{y}+v,-p(s)<y<q(s), s>s_{0}:=-\ln (T) / 2, \\
& v_{y}(-p(s), s)=\gamma_{1}, v_{y}(q(s), s)=\gamma_{2}, s>s_{0}, \\
& v(-p(s), s)=p(s) \tan \beta_{1}, v(q(s), s)=q(s) \tan \beta_{2}, s>s_{0} .
\end{aligned}
$$

Under the assumption (1.25), it is proved in section 4 that $u$ is positive. It is clear that

$$
p(s)>0, q(s)>0 .
$$


Combining the lemmas from section 4 , we have

LEMma 5.1. For a positive solution, we have

$$
\max \left[\left(\tan \beta_{2}\right) y,-\left(\tan \beta_{1}\right) y\right] \leq v(y, s) \leq C,\left|v_{y}(y, s)\right| \leq C,
$$

and

$$
0<p(s) \leq C, \quad 0<q(s) \leq C .
$$

Proof. (5.5) is an immediate result from Lemma 4.6. The gradient bound is an immediate result from the gradient bound for $u$. Lemma 4.3 gives the lower bound estimates for $v(y, s)$.

The estimate $u(0, t) \leq C \sqrt{T-t}$ implies that $v(0, s) \leq C$. Since $p(s), q(s)$ are bounded,

$$
v(y, s) \leq v(0, s)+\left\|v_{y}\right\|_{L^{\infty}} \leq C .
$$

The lemma is proved.

Next, we prove the following nondegeneracy lemma.

Lemma 5.2. There exists $c>0$ such that

$$
p(s)>c, q(s)>c .
$$

Proof. Using Lemma 4.7, we obtain, for some $c_{1}>0$,

$$
v(0, s) \geq c_{1} \text {. }
$$

Using this estimate and the gradient bounds on $v$, we obtain

$$
v(y, s) \geq \frac{c_{1}}{2} \quad \text { for }|y|<c_{2},
$$

for some $c_{2}>0$. Now the lemma follows from the boundary condition.

Using Lemma 5.2 and interior parabolic estimates, all derivatives of $v(y, s)$ are bounded at $y=0$. We now derive the following estimate for the second-order derivative.

Lemma 5.3. There exists a constant $C$ such that

$$
-C \leq v_{y y}(y, s) \leq C e^{-s} \text { for } \quad-p(s) \leq y \leq q(s), s_{0}<s<\infty .
$$

Proof. If we rewrite the estimate in Lemma 2.3, we obtain

$$
v_{y y} \leq \sqrt{2} C e^{-s} \text {. }
$$

Using Lemmas 2.3 and 4.7, we get

$$
u_{x x}(x, t) \geq-\frac{C}{\sqrt{T-t}} .
$$

Rewriting this in the $y$ variable, we get $v_{y y} \geq-C$.

Combining these lemmas and using the boundary conditions, we obtain

LEMMA 5.4.

$$
\left|p^{\prime}(s)\right|+\left|q^{\prime}(s)\right| \leq C \text { for } s_{0}<s<\infty .
$$

Proof. Since $v_{s}$ is bounded, we use the boundary condition to write $p^{\prime}(s)$ and $q^{\prime}(s)$ in terms of $v_{s}$ to conclude the lemma. 
If we let

$$
D^{*}(s)=\int_{-p(s)}^{q(s)} v(y, s) d y-\frac{1}{2} p_{1}^{2}(s) \tan \beta_{1}-\frac{1}{2} p_{2}^{2}(s) \tan \beta_{2}
$$

then

$$
D^{*}(s)=\frac{1}{2(T-t)} D(t)=\frac{1}{2}\left[a\left(\gamma_{1}\right)-a\left(\gamma_{2}\right)\right] \text { for } \quad s_{0}<s<\infty .
$$

We next derive energy estimates.

Following 13] (see also [8]), we define

$$
E(s)=\int_{-p(s)}^{q(s)} \Phi\left(y, v(y, s), v_{y}(y, s)\right) d y,
$$

where $\Phi=\Phi(y, v, w)$ is to be determined. Then

$$
\frac{d}{d s} E(s)=J_{0}+J_{1}+J_{2},
$$

where

$$
\begin{aligned}
J_{0}= & -\int_{-p(s)}^{q(s)} \frac{1}{a^{\prime}\left(v_{y}(y, s)\right)} \Phi_{w w}\left(y, v(y, s), v_{y}(y, s)\right)\left|v_{s}\right|^{2}(y, s) d y, \\
J_{1}= & \Phi\left(q(s), q(s) \tan \beta_{2}, \gamma_{2}\right) q^{\prime}(s)+\Phi\left(-p(s), p(s) \tan \beta_{1}, \gamma_{1}\right) p^{\prime}(s) \\
& +\Phi_{w}\left(q(s), q(s) \tan \beta_{2}, \gamma_{2}\right)\left(\tan \beta_{2}-\gamma_{2}\right) q^{\prime}(s) \\
& -\Phi_{w}\left(-p(s), p(s) \tan \beta_{1}, \gamma_{1}\right)\left(\tan \beta_{1}+\gamma_{1}\right) p^{\prime}(s), \\
J_{2}= & \int_{-p(s)}^{q(s)}\left\{\Phi_{v}-\Phi_{w y}-\Phi_{w v} v_{y}-\Phi_{w w}\left[\frac{y v_{y}-v}{a^{\prime}\left(v_{y}\right)}\right]\right\} v_{s}(y, s) d y \\
\equiv & \int_{-p(s)}^{q(s)} K\left(y, v(y, s), v_{y}(y, s)\right) v_{s}(y, s) d y .
\end{aligned}
$$

Let

$$
\Phi(y, v, w)=\int_{0}^{w}(w-\sigma) P(y, v, \sigma) d \sigma-\int_{0}^{v} \frac{\mu}{a^{\prime}(0)} P(y, \mu, 0) d \mu .
$$

Then

$$
\Phi_{w w}(y, v, w)=P(y, v, w)
$$

and

$$
K(y, v, w)=\int_{0}^{w}\left\{-\sigma \frac{\partial P}{\partial v}(y, v, \sigma)-\frac{\partial P}{\partial y}(y, v, \sigma)-\frac{\partial}{\partial \sigma}\left[P(y, v, \sigma) \frac{y \sigma-v}{a^{\prime}(\sigma)}\right]\right\} d \sigma .
$$

We want to construct $P$ so that the braces $\{\cdots\}$ in the above vanish. Let $P(y, v, w)=$ $\exp (Q(y, v, w))$. Then this is equivalent to

$$
w Q_{v}(y, v, w)+Q_{y}(y, v, w)+\frac{y w-v}{a^{\prime}(w)} Q_{w}(y, v, w)+\frac{\partial}{\partial w}\left\{\frac{y w-v}{a^{\prime}(w)}\right\}=0 .
$$

This linear PDE is solved through characteristics.

We let $\psi(\eta ; y, v, w)$ be defined as the solution of the conjugate problem with the terminal condition:

$$
\begin{aligned}
& a^{\prime}\left(\psi_{\eta}\right) \psi_{\eta \eta}-\eta \psi_{\eta}+\psi=0, \eta \in\left(-C^{*}, y\right), y \leq C^{*} \\
& \psi(y ; y, v, w)=v, \quad \psi_{\eta}(y ; y, v, w)=w,
\end{aligned}
$$


where we assume that $p(s) \leq C^{*}, q(s) \leq C^{*}$ for all $s>s_{0}$. Since $a^{\prime}(s) \in(l, 1 / l)$ for some $l>0$, the solution of this ODE is well defined. It is clear that for bounded $v$ and $w$, the solution $\psi$ and its derivative $\psi_{\eta}$ are uniformly bounded.

Differentiating (5.14) in $y$, we obtain $\psi_{\eta}(y ; y, v, w)+\psi_{y}(y ; y, v, w)=0$, and hence

$$
\psi_{y}(y ; y, v, w)=-\psi_{\eta}(y ; y, v, w)=-w .
$$

Similarly, since $\psi_{\eta \eta}(y ; y, v, w)+\psi_{\eta y}(y ; y, v, w)=0$,

$$
\begin{aligned}
\psi_{y \eta}(y ; y, v, w) & =-\psi_{\eta \eta}(y ; y, v, w) \\
& =\frac{-y \psi_{\eta}(y ; y, v, w)+\psi(y ; y, v, w)}{a^{\prime}\left(\psi_{\eta}(y ; y, v, w)\right)}=\frac{-y w+v}{a^{\prime}(w)} .
\end{aligned}
$$

Next, differentiating (5.14) in $v$ and $w$, respectively, we find that

$$
\psi_{v}(y ; y, v, w)=1, \quad \psi_{v \eta}(y ; y, v, w)=0, \quad \psi_{w}(y ; y, v, w)=0, \quad \psi_{w \eta}(y ; y, v, w)=1 .
$$

Thus the functions $\psi_{y}(\eta ; y, v, w)$ and $-w \psi_{v}(\eta ; y, v, w)+\frac{-y w+v}{a^{\prime}(w)} \psi_{w}(\eta ; y, v, w)$ and their first derivatives in $\eta$ agree at $\eta=y$.

Differentiating (5.13) with respect to the parameters $y, v$ and $w$, using also the uniqueness of the solution of the corresponding ODE, we derive

$$
\psi_{y}(\eta ; y, v, w)=-w \psi_{v}(\eta ; y, v, w)+\frac{-y w+v}{a^{\prime}(w)} \psi_{w}(\xi ; y, v, w) .
$$

Let $R(y, v, w)=\frac{\partial}{\partial w}\left\{\frac{y w-v}{a^{\prime}(w)}\right\}$ and define

$$
Q(y, v, w)=-\int_{0}^{y} R\left(\eta, \psi(\eta, y, v, w), \psi_{\eta}(\eta, y, v, w)\right) d \eta
$$

Then a direct computation shows that (using also (5.15))

$$
\begin{gathered}
w Q_{v}(y, v, w)+Q_{y}(y, v, w)+\frac{y w-v}{a^{\prime}(w)} Q_{w}(y, v, w)+\frac{\partial}{\partial w}\left\{\frac{y w-v}{a^{\prime}(w)}\right\} \\
=\quad w Q_{v}(y, v, w)+\frac{y w-v}{a^{\prime}(w)} Q_{w}(y, v, w)-\int_{0}^{y}\left(R_{v} \psi_{y}+R_{w} \psi_{\eta y}\right) d \eta \\
=-\int_{0}^{y}\left\{w\left(R_{v} \psi_{v}+R_{w} \psi_{\eta v}\right)+\frac{y w-v}{a^{\prime}(w)}\left(R_{v} \psi_{w}+R_{w} \psi_{\eta w}\right)\right. \\
\left.\quad+\left(R_{v} \psi_{y}+R_{w} \psi_{\eta y}\right)\right\} d \eta \\
=-\int_{0}^{y}\left\{R_{v} \cdot\left[w \psi_{v}+\frac{y w-v}{a^{\prime}(w)} \psi_{w}+\psi_{y}\right]\right\} d \eta \\
\quad-\int_{0}^{y}\left\{R_{w} \cdot\left[w \psi_{\eta v}+\frac{y w-v}{a^{\prime}(w)} \psi_{\eta w}+\psi_{\eta y}\right]\right\} d \eta .
\end{gathered}
$$

The first term of the above expressions vanishes by (5.15), and the second term also vanishes by differentiating (5.15) in $\eta$.

Substituting this equality into the expression for $K$, we get

$$
K(y, v, w) \equiv 0,
$$


and hence

$$
J_{2}=0
$$

Using the bounds on $p(s)$ and $q(s)$, we obtain that

$$
\sup _{s_{0}<s<\infty}\left|\int_{s_{0}}^{s} J_{1}(\tau) d \tau\right| \leq C<\infty .
$$

Thus we proved:

THEOREM 5.5.

$$
\int_{s_{0}}^{\infty} \int_{-p(s)}^{q(s)} \frac{1}{a^{\prime}\left(v_{y}(y, s)\right)} P\left(y, v(y, s), v_{y}(y, s)\right)\left|v_{s}\right|^{2}(y, s) d s<\infty .
$$

It is clear that $l \leq a^{\prime}(s) \leq 1 / l$ for some $l>0$, and therefore

$$
c_{0} \leq \frac{P\left(y, v(y, s), v_{y}(y, s)\right)}{a^{\prime}\left(v_{y}(y, s)\right)} \leq C \quad \text { for } \quad s_{0}<s<\infty
$$

for some $0<c_{0}<C<\infty$.

Proof of Theorem 1.5 (i) (ii). We follow the standard procedure in [7, which was already used in the proof of Theorem 1.3

Theorem 5.5. together with the estimates on the derivatives of the solution established in sections 4 and 5, immediately imply that a subsequence will convergence to the $\omega$ limit which is a stationary solution of (5.1). The $v_{y y}$ estimates ensure that the boundary conditions remain in force after taking the limit.

We call a stationary solution of (5.1)-(5.3) a backward self-similar solution. Note that in general we do not have the uniqueness of backward self-similar solutions. For example, in the heat equation case, there are at least two backward self-similar solutions if $\tan ^{-1} G_{c}<\alpha_{2}<-\beta_{1}<\alpha_{1} \leq \beta_{2}$, where $G_{c}<-\tan \beta_{1}$ is a constant depending only on $\alpha_{1}$ and $\beta_{1}$. Also, there is at least one backward self-similar solution if (1.25) holds (cf. Theorem 4.1 in [9]).

Although the ODE solution may not be unique in general, there can only be finitely many solutions in the case that $a(s)$ is analytic. We will prove this fact in section 6 . This fact will enable us to establish the following theorem.

Proof of Theorem 1.5 (iii). Suppose that both $\varphi_{1}$ and $\varphi_{2}$ are the $\omega$-limit. The estimates in the previous sections imply that $\varphi_{i}(i=1,2)$ are well-defined in a small neighborhood of 0 . If $\varphi_{1} \equiv \varphi_{2}$ in a small neighborhood of 0 , then it is clear that $\varphi_{1} \equiv \varphi_{2}$ everywhere, by uniqueness of the ODE. Thus if the $\omega$-limit is not unique, then there must exists a small $\delta$ such that $\varphi_{1}(\delta) \neq \varphi_{2}(\delta)$. For any number $\eta$ between $\varphi_{1}(\delta)$ and $\varphi_{2}(\delta)$, we can choose $s_{j} \rightarrow \infty$ such that $v\left(\delta, s_{j}\right)=\eta$. Using the compactness and the Lyapunov function we find that $v\left(y, s+s_{j}\right)$ will converge to the ODE solution which is an $\omega$-limit with value $\eta$ at $y=\delta$. Thus we obtain a continuum of $\omega$-limits, which is a contradiction to the result in the next section.

6. The backward self-similar solution. The backward self-similar solution in the case of the heat equation $(a(s) \equiv s)$ has been studied in $[9]$. 
For the general case, the limit $\varphi$ will satisfy:

$$
\begin{aligned}
& {\left[a\left(\varphi^{\prime}\right)\right]^{\prime}-y \varphi^{\prime}+\varphi=0,-p<y<q, \quad p>0, q>0,} \\
& \varphi^{\prime}(-p)=\gamma_{1}, \varphi^{\prime}(q)=\gamma_{2}, \\
& \varphi(-p)=p \tan \beta_{1}, \quad \varphi(q)=q \tan \beta_{2} .
\end{aligned}
$$

Lemma 6.1. Under the assumption (1.25), there exists $\varepsilon_{0}>0$ such that (6.1)-(6.3) has no solution for $p \in\left[0, \varepsilon_{0}\right]$.

Proof. We let $\varphi(y, p)$ be the solution of

$$
\begin{aligned}
& {\left[a\left(\varphi_{y}\right)\right]_{y}-y \varphi_{y}+\varphi=0, y>-p} \\
& \varphi_{y}(y, p)=\gamma_{1} \text { for } y=-p \\
& \varphi(y, p)=p \tan \beta_{1} \text { for } y=-p .
\end{aligned}
$$

It is clear that if $p=0$, then $\varphi(y, 0)=\gamma_{1} y$ is the unique solution of (6.4)-(6.6) and therefore the second equality in (6.2) cannot be satisfied. For $p>0$, let $G(y, p)=$ $y \varphi_{y}(y, p)-\varphi(y, p)$. Then

$$
G_{y}=\frac{y}{a^{\prime}\left(\varphi_{y}(y, p)\right)} G, \quad G(-p, p)=-p\left(\gamma_{1}+\tan \beta_{1}\right)
$$

Thus

$$
G(y, p)=-p\left(\gamma_{1}+\tan \beta_{1}\right) \exp \int_{-p}^{y} \frac{\tau}{a^{\prime}\left(\varphi_{y}(\tau, p)\right)} d \tau<0
$$

and

$$
\lim _{y \rightarrow+\infty} G(y, p)=-\infty .
$$

In particular, $\varphi_{y y}(y, p)=G / a^{\prime}\left(\varphi_{y}\right)<0$, and $\varphi_{y}(y, p)<\gamma_{1}$ for $y>-p$. Thus if a solution of (6.1) $-(6.3)$ exists, then

$$
q \tan \beta_{2}=\varphi(q, p) \leq \varphi(-p, p)+\gamma_{1}(q+p)=p \tan \beta_{1}+\gamma_{1}(q+p),
$$

which implies that $q\left(\tan \beta_{2}-\gamma_{1}\right) \leq p\left(\tan \beta_{1}+\gamma_{1}\right)$. From (6.7) and the relationship $\varphi_{y y}(y, p)=G / a^{\prime}\left(\varphi_{y}\right)$, we also obtain the estimates for $\varphi_{y y}$. Combining all these estimates, we obtain

$$
\gamma_{1}-\gamma_{2}=-\int_{-p}^{q} \varphi_{y y}(\tau, p) d \tau \leq(p+q) \max _{\tau \in[-p, q]}\left(-\varphi_{y y}(\tau, p)\right) \leq C \varepsilon_{0}^{2} \quad \text { for } \quad p \in\left(0, \varepsilon_{0}\right] .
$$

This is a contradiction if $\varepsilon_{0}$ is small.

We next prove:

Lemma 6.2. In addition to the assumption (1.25), we assume that $a(s)$ is analytic in $s$ for $s \in\left[\gamma_{2}, \gamma_{1}\right]$. Then there are at most finitely many solutions of $(p, q)$ satisfying (6.1) -6.3 .

Proof. For $p>\varepsilon_{0}$, the $-\varphi_{y y}(y, p)$ is bounded from below by a positive constant. Thus we can uniquely solve $q=q(p)>-p$ such that

$$
\varphi_{y}(q(p), p) \equiv \gamma_{2} .
$$


The estimates (6.8) imply that $q(p)$ is bounded from above. It is also clear that $\gamma_{2} \leq$ $\varphi_{y} \leq \gamma_{1}$ for $-p \leq p \leq q(p)$.

Define, for $p \geq \varepsilon_{0}$,

$$
K(p):=\varphi(q(p), p)-q(p) \tan \beta_{2} .
$$

The above discussion implies that $K(p)$ is well defined for all $p \geq \varepsilon_{0}$.

If $p_{0}$ is a zero for $K(p)$, we want to show $p_{0}$ is an isolated zero for $K$.

If $a(s)$ is analytic for $s \in\left[\gamma_{2}, \gamma_{1}\right]$, then $K$ is analytic. Therefore the zeros of $K$ cannot have a limit point unless $K(p)$ is identically zero. Thus if $p_{0}$ is not an isolated zero for $K$, then $K(p) \equiv 0$ for $p \geq \varepsilon_{0}$. However, Lemma 6.1 implies that $K\left(\varepsilon_{0}\right) \neq 0$. Thus we obtain a contradiction and we proved that the zeros of $K$ are isolated.

If $p$ is sufficiently large, then it is clear from (6.7) that $q(p)<0$ and thus $K(p)>0$. Since $K(p)$ can only have finitely many isolated zeros on a bounded interval, the lemma is proved.

Acknowledgment. The authors would like to thank the referee for helpful suggestions. This work was partially completed during a visit of the second author to the National Taiwan Normal University. This visit was sponsored by NSC 92-2735-M-001001. He would like to express his gratitude for the hospitality during the visit.

\section{REFERENCES}

[1] J. D. Buckmaster and G. S. S. Ludford, Theory of Laminar Flames, Cambridge University Press, Cambridge, 1982. MR0666866 (84f:80011)

[2] L. A. Caffarelli and J. L. Vazquez, A free-boundary problem for the heat equation arising in flame propagation, Trans. Amer. Math. Soc. 347 (1995), 411-441. MR1260199 (95e:35097)

[3] Y.-L. Chang, J.-S. Guo, and Y. Kohsaka, On a two-point free boundary problem for a quasilinear parabolic equation, Asymptotic Analysis 34 (2003), 333-358. MR.1993377 (2005a:35288)

[4] H.-H. Chern, J.-S. Guo and C.-P. Lo, The self-similar expanding curve for the curvature flow equation, Proc. Amer. Math. Soc. 131 (2003), 3191-3201. MR1992860 (2004f:35193)

[5] A. Friedman, Partial Differential Equations of Parabolic Type, Prentice-Hall, Englewood Cliffs, NJ, 1964. MR0181836 (31:6062)

[6] V. A. Galaktionov, J. Hulshof and J. L. Vazquez, Extinction and focusing behaviour of spherical and annular flames described by a free boundary problem, J. Math. Pures Appl. 76 (1997), 563-608. MR.1472115 (98h:35238)

[7] Y. Giga and R. V. Kohn, Asymptotically self-similar blow-up of semilinear heat equations, Comm. Pure and Applied Math. 38 (1985), 297-319. MR0784476 (86k:35065)

[8] J.-S. Guo, and B. Hu, Quenching profile for a quasilinear parabolic equation, Quarterly of Applied Math. 58 (2000), 613-626. MR1788421 (2001m:35155)

[9] J.-S. Guo and Y. Kohsaka, Self-similar solutions of two-point free boundary problem for heat equation, Nonlinear Diffusive Systems and Related Topics, RIMS Kokyuroku 1258, Research Institute for Mathematical Sciences, Kyoto University, April, 2002, pp. 94-107. MR1927223

[10] D. Hilhorst and J. Hulshof, A free boundary focusing problem, Proc. Amer. Math. Soc. 121 (1994), 1193-1202. MR1233975 (94j:35200)

[11] Y. Kohsaka, Free boundary problem for quasilinear parabolic equation with fixed angle of contact to a boundary, Nonlinear Analysis 45 (2001), 865-894. MR.1845031 (2002j:35320)

[12] J. L. Vazquez, The free boundary problem for the heat equation with fixed gradient condition, Free boundary problems, theory and applications, Zakopane, Poland (1995), Pitman Res. Notes in Math. Ser. 363, 277-302. MR 1462990 (98h:35246)

[13] T. I. Zelenjak, Stabilization of solutions of boundary value problems for a second-order parabolic equation with one space variable, Differential Equations 4 (1968), 17-22. MR0223758 (36:6806) 